\title{
DIAGNÓSTICOS E INTERVENÇÕES DE ENFERMAGEM A CRIANÇAS COM SINAIS RESPIRATÓRIOS DE GRAVIDADE DA COVID-19
}

\author{
NURSING DIAGNOSES AND INTERVENTIONS \\ FOR CHILDREN WITH RESPIRATORY SIGNS OF \\ COVID-19 SEVERITY
}

\section{DIAGNÓSTICOS E INTERVENCIONES DE ENFERMERÍA PARA NIÑOS CON SIGNOS RESPIRATORIOS DE GRAVEDAD COVID-19}

\author{
Renise Bastos Farias Dias ${ }^{1}$ \\ Luana Cavalcante Costa Ferraz ${ }^{2}$ \\ Nirliane Ribeiro Barbosa ${ }^{3}$ \\ Rita de Cássia Batista de Oliveira Peixoto ${ }^{4}$ \\ Maria Betânia Monteiro de Farias \\ Larissa Tenório Andrade Correia ${ }^{6}$ \\ Andrey Ferreira da Silva ${ }^{7}$
}

Como citar este artigo: Dias RBF, Ferraz LCC, Barbosa NR, Peixoto RCBO, Farias MBM, Correia LTA, et al. Diagnósticos e intervenções de enfermagem a crianças com sinais respiratórios de gravidade da COVID-19. Rev baiana enferm. 2021;35:e37467.

\begin{abstract}
Objetivo: descrever diagnósticos e intervenções de enfermagem a crianças com sinais respiratórios de gravidade da COVID-19. Método: estudo exploratório, descritivo, do tipo revisão narrativa, realizado em junho de 2020, embasado pelas evidências científicas publicadas na base PUBCOVID-19, para posterior elaboração de diagnósticos utilizando a Taxonomia North American Nursing Diagnoses Association - International, e intervenções ancoradas na Nursing Interventions Classification. Resultados: a dispneia e o desconforto respiratório foram os sinais de gravidade mais evidenciados na criança com COVID-19. Foram elaboradas três principais afirmativas diagnósticas: Troca de Gases Prejudicada, Padrão Respiratório Ineficaz e Ventilação Espontânea Prejudicada, com 24 Intervenções de Enfermagem correspondentes em quatro domínios: o fisiológico, comportamental, de segurança, e o de família. Conclusão: a descrição dos diagnósticos e intervenções de enfermagem, ancorados pelas taxonomias NANDA e NIC, respectivamente, apresenta inovação na literatura científica brasileira.
\end{abstract}

Descritores: Enfermagem Pediátrica. Cuidados de Enfermagem. Infecções por Coronavírus. Diagnóstico de Enfermagem.

\footnotetext{
Enfermeira. Mestre em Enfermagem. Docente da Universidade Federal de Alagoas. Arapiraca, Alagoas, Brasil. http://orcid.org/0000-0003-0960-9034. Enfermeira. Mestre em Enfermagem. Docente da Universidade Federal de Alagoas. Arapiraca, Alagoas, Brasil. https://orcid.org/0000-0002-6532-4642. Enfermeira. Mestre em Enfermagem. Docente da Universidade Federal de Alagoas. Arapiraca, Alagoas, Brasil. http://orcid.org/0000-0002-1737-555X. Enfermeira. Mestre em Ciências. Docente da Universidade Federal de Alagoas. Arapiraca, Alagoas, Brasil. http://orcid.org/0000-000 I-9891-6188.

Enfermeira. Mestre em Enfermagem. Docente da Universidade Federal de Alagoas. Arapiraca, Alagoas, Brasil. http://orcid.org/0000-0002-8339-9525.

Enfermeira. Mestre em Nutrição Humana. Docente da Universidade Federal de Alagoas. Arapiraca, Alagoas, Brasil. http://orcid.org/0000-000 I-5932-628I .

Enfermeiro. Doutor em Enfermagem. Docente da Universidade Federal de Alagoas. Arapiraca, Alagoas, Brasil. silva.andreyl99|@hotmail.com. http://orcid. org/0000-0002-1038-7443.
} 
Objective: to describe nursing diagnoses and interventions to children with respiratory signs of COVID-19 severity. Method: exploratory, descriptive, narrative reviewstudy, conducted in June 2020, based on scientific evidencepublished in the PUBCOVID-19 database, for further preparation of diagnoses using the North American Nursing Diagnoses Association - International Taxonomy, and interventions anchored in the Nursing Interventions Classification. Results: dyspnea and respiratory distress were the most evident signs of severity in children with COVID-19. Three main diagnostic statements were elaborated: Impaired Gas Exchange, Ineffective Respiratory Pattern and Impaired Spontaneous Ventilation, with 24 Nursing Interventions corresponding in four domains: physiological, behavioral, safety, and family. Conclusion: the description of nursing diagnoses and interventions, anchored by NANDA and NIC taxonomies, respectively, presents innovation in the Brazilian scientific literature.

Descriptors: Pediatric Nursing. Nursing Care. Coronavirus infections. Nursing Diagnosis.

Objetivo: describir diagnósticos e intervenciones de enfermería a niños con signos respiratorios de gravedad COVID-19. Método: estudio exploratorio, descriptivo, de revisión narrativa, realizado en junio de 2020, basado en evidencia científica publicada en la base de datos PUBCOVID-19, para la preparación ulterior de diagnósticos utilizando la Taxonomía North American Nursing Diagnosis Association - International, e intervenciones ancladas en la Clasificación de Intervenciones de Enfermería. Resultados: la disnea y la dificultad respiratoria fueron los signos más evidentes de gravedad en niños con COVID-19. Se elaboraron tres declaraciones diagnósticas principales: Deterioro de Intercambio de Gases, Patrón Respiratorio Ineficaz y Deterioro de Ventilación Espontánea, con 24 Intervenciones de Enfermería correspondientes en cuatro ámbitos: fisiológica, conductual, seguridad y familia. Conclusión: la descripción de los diagnósticos e intervenciones de enfermería, anclados por taxonomías NANDA y NIC, respectivamente, presenta innovación en la literatura científica brasileña.

Descriptores: Enfermería Pediátrica. Atención de Enfermería. Infeccionespor Coronavirus. Diagnóstico de Enfermería.

\section{Introdução}

A Coronavirus Disease-19 (COVID-19) é uma doença recém-identificada, causada pelo Severe Acute Respiratory Syndrome Coronavirus 2 $(\mathrm{SARS}-\mathrm{CoV}-2)^{(1)}$. Trata-se de um vírus de Ácido Ribonucleico (RNA) pertencente ao grupo da família dos Coronaviridae, que apresenta elevada taxa de transmissibilidade e é responsável pelo crescente número de casos de infecções respiratórias em todo o mundo ${ }^{(2-3)}$.

Em crianças, essa doença manifesta-se por meio de sinais clínicos clássicos, como febre acima de $37,8{ }^{\circ} \mathrm{C}$, tosse, dor de $\operatorname{garganta}^{(4)}$ ou mesmo por outros sintomas, como disfunções respiratórias, distúrbios gastrointestinais ou neurológicos, choque e síndrome inflamatória multissistêmica $^{(5)}$. Embora as crianças sejam comumente acometidas por infecções do trato respiratório ${ }^{(6)}$, estudos têm mostrado que as crianças representam 1 a 5\% dos casos de COVID-19 diagnosticados $^{(7)}$. Nos Estados Unidos, 1,7\% dos casos de pacientes com COVID-19 tinha menos de 18 anos, enquanto na China continental e na Itália, esse número correspondia a 1,3\% dos $\operatorname{casos}^{(8-9)}$. No Brasil, segundo o Boletim Epidemiológico n. 17, de maio de 2020, foram notificados 347.398 casos de COVID-19 na população em geral; destes, 662 (5,25\%) crianças de
0 a 12 anos incompletos foram notificadas com Síndrome Respiratória Aguda Grave (SRAG) confirmado para COVID-19 ${ }^{(10)}$.

Embora as evidências demonstrem que a maioria das crianças com a COVID-19 são assintomáticas ou têm um curso clínico da doença menos agressivo ${ }^{(11)}$, novos achados indicam a possibilidade de complicações que levariam ao agravamento da doença ${ }^{(12)}$. No Brasil, de 219 crianças com SRAG que necessitaram de internação em Unidade de Tratamento Intensivo (UTI), 159 receberam suporte ventilatório e 62 avançaram para óbito ${ }^{(10)}$. Estudo realizado no Centro Chinês de Controle e Prevenção de Doenças demonstrou que, das 731 crianças com virologia confirmada para COVID-19, 12,9\% eram assintomáticos, 43,1\% casos leves, 41\% moderados, 2,5\% graves e 0,4\% casos críticos, demonstrando o processo de evolução da doença e a necessidade de maior atenção e cuidado a essa população ${ }^{(13)}$.

Diante da magnitude evidenciada, e entendendo que se faz necessário o acompanhamento da progressão da doença com identificação de sinais e sintomas de risco para gravidade, para que os processos de cuidado sejam sistematizados, os fluxogramas de atenção à saúde mostram-se relevantes por consolidar as principais evidências da literatura, além de auxiliarem na 
otimização do processo de cuidado. Entretanto, precisam ser claros aos diferentes processos de cuidado à saúde ${ }^{(14)}$, sobretudo no contexto de uma nova doença, com manifestações clínicas distintas. Para respaldar técnica e cientificamente os profissionais de saúde no Brasil em suas respectivas condutas frente à criança com sinais de gravidade de SARS-Cov-2, o Ministério da Saúde (MS) elaborou e publicou, em março de 2020, o fluxo de manejo clínico pediátrico na Atenção Especializada $^{(15)}$.

Diante desses sintomas de gravidade da criança com infecção pelo Coronavírus SARS-CoV-2, urge a necessidade da execução de um cuidado adequado à recuperação das crianças. Nesse contexto, os(as) profissionais de enfermagem têm um relevante papel no processo de cuidar da criança com COVID-19, embasado nas evidências científicas, cuja intervenção de enfermagem seja individualizada e eficaz ${ }^{(16)}$. Nesse sentido, faz-se necessário a compreensão dos possíveis diagnósticos de enfermagem para uma efetiva elaboração das intervenções que podem ser aplicadas, otimizando e qualificando as práticas assistenciais da(o) enfermeira(o) ${ }^{(17)}$.

A elaboração de diagnósticos e intervenções de enfermagem são essenciais para o processo de trabalho da(o) enfermeira(o), pois proporcionam elementos indispensáveis para uma assistência sistematizada. Além disso, esses fazem parte do processo de enfermagem, facilitando a organização e a implementação do cuidado de enfermagem, indispensável diante das situações de pandemia ${ }^{(18)}$. Diante desse cenário, levantamos a seguinte questão: Quais são os diagnósticos e intervenções de enfermagem a crianças com sinais respiratórios de gravidade da COVID-19?

Portanto, o presente estudo tem como objetivo descrever afirmativas de diagnósticos e de intervenções de enfermagem a crianças com sinais respiratórios de gravidade da COVID-19.

\section{Método}

Trata-se de estudo exploratório, descritivo, do tipo revisão narrativa, que permite compreender e estabelecer relações entre produções em um determinado contexto, sinalizando aspectos recorrentes e novas perspectivas ${ }^{(19)}$. A busca de dados foi realizada em documentos oficiais brasileiros e artigos científicos que versam sobre os sinais de gravidade de SARS-CoV-2, COVID-19, em crianças.

Para o alcance do objetivo proposto nesta pesquisa foram percorridos dois momentos:

a) identificação dos sinais de gravidade de SARS-CoV-2, COVID-19, presentes no fluxo de manejo clínico pediátrico preconizado pelo $\mathrm{MS}^{(15)}$, seguida de busca, no mês de junho de 2020, de evidências científicas na base de dados PUBCOVID19, frente a sua representatividade na consolidação de informações científicas nacionais e internacionais;

b) construção de afirmativas diagnósticas e de intervenções de enfermagem, com foco na população pediátrica com sinais respiratórios de gravidade da COVID-19.

Foram analisados o fluxo de manejo clínico pediátrico na Atenção Especializada COVID-19, publicado pelo Ministério da Saúde, uma vez que se trata de documento norteador da condução clínica desses casos no contexto brasileiro ${ }^{(15)}$, as notas publicadas pela Sociedade Brasileira de Enfermeiros Pediatras e da Sociedade Brasileira de Pediatria, órgãos de referência na área de pediatria no Brasil, além de artigos selecionados em base de dados da COVID-19. Em todas as buscas foram considerados os sinais de gravidade para a Síndrome Respiratória Aguda Grave em pediatria, sendo selecionado para a discussão, dentre os sinais presentes no Fluxo de Manejo Pediátrico, o mais relevante. De acordo com as evidências científicas, os sinais respiratórios de gravidade são os mais evidenciados na literatura ${ }^{(20-21)}$.

Após a seleção dos sinais respiratórios de gravidade, buscou-se elaborar os Diagnósticos de Enfermagem (D.E.), definidos como julgamento, frente ao fenômeno apresentado pelo indivíduo ou pela coletividade, que precisam ter um sentido clínico e uma taxonomia com linguagem 
universal $^{(21)}$.ParaaformulaçãodosD.E., utilizou-se a taxonomia North American Nursing Diagnosis Association - International (NANDA-I) ${ }^{(22)}$. As Características Definidoras foram selecionadas na NANDA-I para justificar a elaboração dos diagnósticos.

Para os diagnósticos elaborados foram selecionadas Intervenções de Enfermagem, entendida como "[...] tratamento, baseado no julgamento e no conhecimento clínico, que seja realizado por uma/um enfermeira(o) para melhorar os resultados do paciente/cliente" ${ }^{\text {(23:20). }}$ Os títulos das intervenções selecionadas foram ancorados na Nursing Interventions Classification (NIC) ${ }^{(24)}$.

Os achados foram organizados em um quadro e analisados à luz da literatura científica nacional e internacional que versa sobre o cuidado à criança, de modo a trazer elementos que justifiquem a definição dos domínios utilizados na construção dos títulos de intervenções de enfermagem selecionados no presente estudo, e possam embasar a tomada de decisão da(o) enfermeira(o) no contexto de cuidado à criança com sinais respiratórios de gravidade da COVID-19.

\section{Resultados}

Tendo em vista os principais sinais de gravidade da SRAG, "dispneia" e "desconforto respiratório", em crianças com COVID-19, foi possível selecionar as características definidoras utilizando a taxonomia da NANDA-I, o que resultou na elaboração de três afirmativas diagnósticas. Em seguida, com base na taxonomia NIC, foram selecionados os títulos de intervenções de enfermagem correspondentes a cada diagnóstico.
No Quadro 1, é possível observar as características definidoras e os Diagnósticos de Enfermagem NANDA-I para pacientes pediátricos com sinais de gravidade para COVID-19, com foco na Classe 4 - Respostas Cardiovasculares/Pulmonares. Os diagnósticos elaborados pertencem a dois domínios, isto é, de duas áreas de interesse para a enfermagem, a saber: Domínio 3 - Eliminação/troca. Diagnóstico: Troca de gases prejudicada. Domínio 4 - Atividade/repouso. Diagnósticos: Padrão Respiratório Ineficaz; Ventilação Espontânea Prejudicada.

Em seguida, foram elaboradas 24 Intervenções de Enfermagem, pela Taxonomia NIC (Quadro 1), localizadas em 4 Domínios:

a) Domínio 2: Fisiológico Complexo, contemplando a Classe G - Controle Eletrolítico e Ácido-Básico, com uma Intervenção; a Classe $\mathrm{H}$ - Controle de Medicamentos, com uma Intervenção; a Classe I - Controle Neurológico, com uma Intervenção e a Classe $\mathrm{K}$ - Controle Respiratório, com dez Intervenções;

b) Domínio 3: Comportamental, contemplando a Classe R - Assistência ao Enfrentamento, com duas Intervenções; Classe S - Educação ao Paciente, com duas Intervenções e a Classe T - Promoção do Conforto Psicológico, com uma Intervenção;

c) Domínio 4: Segurança, contemplando a Classe V - Controle de Risco, com cinco Intervenções;

d) Domínio 5: Família, contemplando a Classe X - Cuidados ao Longo da Vida, com uma Intervenção. 
Quadro 1 - Descrição das características definidoras aos Diagnósticos de Enfermagem (NANDA-I) Troca de Gases Prejudicada, Padrão Respiratório Ineficaz e Ventilação Espontânea Prejudicada, seguida dos Títulos das Intervenções de Enfermagem (NIC) correspondentes, com foco em pacientes pediátricos com sinais de gravidade para COVID-19

\begin{tabular}{|c|c|c|}
\hline $\begin{array}{l}\text { CARACTERÍSTICAS } \\
\text { DEFINIDORAS }\end{array}$ & \begin{tabular}{|c} 
DIAGNÓSTICOS \\
DE ENFERMAGEM \\
North American \\
Nursing Diagnosis \\
Association \\
\end{tabular} & $\begin{array}{c}\text { TÍTULOS DE INTERVENÇÕES DE } \\
\text { ENFERMAGEM Nursing Interventions } \\
\text { Classification }\end{array}$ \\
\hline $\begin{array}{l}\text {-Dispneia } \\
\text {-Batimento da asa de } \\
\text { nariz } \\
\text {-Capacidade vital } \\
\text { diminuída } \\
\text {-Uso da musculatura } \\
\text { acessória para respirar } \\
\text {-Excursão torácica } \\
\text { alterada } \\
\text {-Padrão respiratório } \\
\text { anormal } \\
\text {-Diminuição da } \\
\text { saturação arterial de } \\
\text { oxigênio (SaO) } \\
\text {-Cooperação diminuída } \\
\text {-Uso aumentado da } \\
\text { musculatura acessória }\end{array}$ & $\begin{array}{l}\text { Troca de Gases } \\
\text { Prejudicada } \\
\text { (Cód.00030) } \\
\\
\text { Padrão Respiratório } \\
\text { Ineficaz (Cód.00032) } \\
\\
\text { Ventilação } \\
\text { Espontânea } \\
\text { Prejudicada } \\
\text { (Cód.00033) }\end{array}$ & $\begin{array}{l}\text {-Monitoração ácido-básico (Cód. 2G-1920) } \\
\text {-Controle de medicamentos (Cód. 2H-2380) } \\
\text {-Monitoração neurológica (Cód. 2I-2620) } \\
\text {-Controle das vias aéreas (Cód. 2K-3140) } \\
\text {-Aspiração das vias aéreas (Cód. 2K-3160) } \\
\text {-Controle das vias aéreas artificiais (Cód. 2K-3180) } \\
\text {-Fisioterapia respiratória (Cód. 2K-3230) } \\
\text {-Controle da Ventilação mecânica: invasiva (Cód. } \\
\text { 2K-3300) } \\
\text {-Controle da Ventilação mecânica: não invasiva } \\
\text { (Cód. 2K-3302) } \\
\text {-Desmame da ventilação mecânica (Cód. 2K-3310) } \\
\text {-Oxigenoterapia (Cód. 2K-3320)* } \\
\text {-Monitoração respiratória (Cód. 2K-3350** } \\
\text {-Assistência ventilatória (Cód. 2K-3390) } \\
\text {-Orientação antecipada (Cód. 3R-5210) } \\
\text {-Apoio emocional (Cód. 3R-5270)* } \\
\text {-Ensino: processo de doença (Cód. 3S-5602) } \\
\text {-Ensino: procedimento/ tratamento (Cód. 3S-5618) } \\
\text {-Redução da ansiedade (Cód. 3T-5820) } \\
\text {-Controle ambiental: segurança (Cód. 4V-6486)* } \\
\text {-Controle ambiental: segurança do trabalhador (Cód. } \\
\text { 4V-6489) } \\
\text {-Controle de infecção (Cód. 4V-6540) } \\
\text {-Supervisão (Cód. 4V-6650)* } \\
\text {-Monitoração dos sinais vitais (Cód. 4V-6680)* } \\
\text {-Apoio familiar (Cód. 5X-7140) }\end{array}$ \\
\hline
\end{tabular}

Fonte: Elaboração própria.

*Intervenções de enfermagem apresentadas pela NIC como essenciais para a especialidade enfermagem pediátrica.

\section{Discussão}

O Fluxo de Manejo Pediátrico do Ministério da Saúde Coronavírus COVID-19, publicado em março de 2020, apresenta como sinais de gravidade a serem observados: dispneia, desconforto respiratório, saturação de oxigênio menor que 95\%, piora nas condições clínicas de doença preexistente e palidez cutânea ${ }^{(15)}$. A literatura apresenta a dispneia e a dificuldade para respirar como os mais citados em pesquisas com crianças, sintomas estes também verificados frequentemente em pacientes na China, por exemplo $^{(24-26)}$. A Sociedade Brasileira de Pediatria acrescenta como sinais para classificar o quadro grave da criança com COVID-19, de acordo com nota publicada em abril de 2020, a incapacidade de amamentar ou beber, letargia ou convulsões $^{(27)}$ - sinais não descritos no Fluxo de Manejo Pediátrico.

Reconhecendo os sinais respiratórios de gravidade presentes no Fluxo de Manejo Pediátrico e na literatura, cabe à enfermeira interpretá-los, para buscar entender as reais necessidades 
dos pacientes. A busca da resolutividade do problema por meio de ações específicas pela(o) enfermeira(o), requer a elaboração de diagnósticos que sejam úteis no planejamento das intervenções que ajudem na qualificação da assistência às crianças ${ }^{(28)}$. Para isso, a identificação de Características Definidoras é necessária para que a(o) enfermeira(o) possa construir diagnósticos individualizados, que superem as prescrições oriundas de protocolos com foco em modelos que reduzam as necessidades humanas básicas ${ }^{(17)}$. Em geral, para que um diagnóstico de enfermagem seja seguramente confirmado, faz-se necessário reconhecer um conjunto de características definidoras, e não somente um único dado clínico, que possa ser relacionado a possíveis diagnósticos voltados à situação clínica específica ${ }^{(29)}$.

Dessa forma, analisando a NANDA-I, identificou-se que as características definidoras, dispneia, batimento da asa de nariz, capacidade vital diminuída, uso da musculatura acessória para respirar, excursão torácica alterada, padrão respiratório anormal, diminuição da saturação arterial de oxigênio e cooperação diminuída, justificam a construção de três afirmativas diagnósticas de enfermagem, voltados aos sinais respiratórios de gravidade. A identificação de diagnósticos de enfermagem a pessoas portadoras de afecções do trato respiratório é particularmente importante e prioritária, pois afetam diretamente uma função vital ${ }^{(29)}$.

O D.E. "Troca de Gases Prejudicada" é definido como excesso ou déficit na oxigenação e/ou eliminação de dióxido de carbono na membrana alveolocapilar ${ }^{(22)}$. A preocupação da(o) enfermeira(o) com a troca de gases em pediatria com sinais respiratórios de gravidade pode levar a esse diagnóstico ${ }^{(22)}$. Fatores como desequilíbrio na ventilação e perfusão, no estado respiratório e nos sinais vitais, podem estar relacionados ao diagnóstico. O D.E. "Padrão Respiratório Ineficaz" é definido como inspiração e/ou expiração que não proporciona ventilação adequada ${ }^{(22)}$. Tal D.E., pode ter como fator relacionado a dor, a ansiedade e a fadiga respiratória, e como possível condição associada à imaturidade neurológica e ao prejuízo neurológico, conforme descrito na NANDA-I. Já o D.E. "Ventilação Espontânea Prejudicada" tem como definição a incapacidade de iniciar e/ou manter respiração independente e adequada para sustentação da vida ${ }^{(22)}$. Adicionalmente, a NANDA-I traz que a fadiga da musculatura respiratória pode ser considerada um fator relacionado e a alteração no metabolismo, uma condição associada, que podem estar presentes em crianças com sinais respiratórios de gravidade para COVID-19 ${ }^{(30)}$.

O D.E. "Padrão Respiratório Ineficaz" sugere Intervenções de Enfermagem voltadas para pacientes pediátricos graves, que não receberam suporte invasivo de oxigênio, enquanto que o diagnóstico Ventilação Espontânea Prejudicada, assim como a Troca de Gases Prejudicada, favorecem intervenções voltadas sobretudo ao paciente grave em ventilação mecânica ${ }^{(31)}$. As duas condições elencadas neste estudo podem ser observadas em pacientes pediátricos com SRAG, como demonstrado em estudo norte-americano, que caracterizou, por meio de uma coorte retrospectiva, realizada com nove crianças hospitalizadas pela COVID-19, que cerca de $89 \%$ das crianças que estavam em estado grave requereram algum suporte respiratório, e cerca de 44\% necessitaram de ventilação mecânica ${ }^{(21)}$.

A literatura científica revela que a utilização de ventilação mecânica invasiva foi evidenciada principalmente em casos de crianças que apresentavam alguma doença preexistente como hidronefrose, leucemia, encefalopatia ou cardiopatia congênita, e que evoluíram com o curso mais grave da COVID-19 ou chegaram a óbito. Essa possível associação também foi verificada em estudo de revisão sistemática e metanálise que buscou levantar todos os aspectos compreendidos até o momento, por meio de 46 materiais científicos que relataram 551 casos de COVID-19 em crianças confirmados laboratorialmente, publicados em países como China, Malásia, Itália, Espanha e Estados Unidos ${ }^{(32)}$. Tais achados convergem com o estudo retrospectivo, realizado em uma unidade de terapia intensiva pediátrica de um hospital de Paris, com 27 crianças admitidas com o diagnóstico confirmado laboratorialmente 
ou altamente suspeito por achados radiológicos, em que buscou-se descrever formas graves de coronavírus, evidenciando que das 19 que apresentavam doenças preexistentes, 9 fizeram uso de ventilação mecânica ${ }^{(33)}$.

Com base nas evidências científicas relatadas, foi possível elencar 24 propostas de Intervenções de Enfermagem NIC, compreendidas em 4 domínios da prática da(o) enfermeira(o), fisiológico complexo, segurança, comportamental e família, cada qual abrangendo suas classes. Tanto os domínios quanto as classes da taxonomia da NIC ajudam as(os) enfermeiras(os) a localizarem e selecionarem as intervenções mais adequadas para os pacientes. O nível de domínio ajuda o profissional a fazer a primeira escolha para a intervenção, e as classes proporcionam um foco maior no cuidado a ser prestado ${ }^{(23)}$. Cada título de intervenção descrito na NIC apresenta de 10 a 30 atividades de enfermagem. Com base nessas atividades, a(o) enfermeira(o) elenca os cuidados a serem prestados, de forma individualizada, a cada criança ou sua família, podendo adicionar novas atividades, se assim o desejar ${ }^{(34)}$. De acordo com a NIC, das 24 propostas de Intervenções apresentadas nesse estudo, 23 necessitam ser realizadas pela(o) enfermeira(o) assistente; e somente a intervenção Redução da Ansiedade pode ser realizada pelo profissional com nível de formação auxiliar ou técnico de enfermagem $^{(23)}$.

No contexto do "domínio fisiológico complexo", considerou-se a escolha pela Classe Controle Neurológico, uma vez que a infecção pelo SARS-CoV-2 em crianças podem manifestar alterações neurológicas, musculoesqueléticas e do nível de consciência. O dano neurológico da invasão potencial do SARS-CoV-2 pode ser responsável parcialmente pela insuficiência respiratória, como vislumbrado em estudo chinês, já que o tronco cerebral e os sinais sensoriais neuronais do sistema nervoso central, controlam de forma coordenada a frequência, a profundidade e o padrão da respiração, com a contração do músculo diafragma e de músculos da caixa torácica, do abdome e das estruturas circundantes $^{(35)}$.
Tendo em vista que a COVID-19 é uma doença nova, e que, até o presente momento, não existem medicamentos de uso autorizado fundamentados em evidências científicas para serem utilizados no seu tratamento ${ }^{(27)}$, a Classe Controle de Medicamentos torna-se relevante no processo de cuidados de enfermagem à criança com sinais respiratórios de gravidade, para possibilitar a intervenção direcionada ao uso seguro e eficaz de medicamentos.

O Controle Respiratório também está presente no domínio fisiológico. Entendendo que a criança com COVID-19, classificada como grave, apresenta necessidades psicobiológicas de oxigenação, caracterizada sobretudo por sinais como dispneia, desconforto respiratório, saturação de oxigênio menor que $95 \%{ }^{(15)}$, esse domínio torna-se essencial. Nesse sentido, duas intervenções são consideradas pela NIC como essenciais para a especialidade enfermagem pediátrica, a Monitoração Respiratória e a Oxigenoterapia ${ }^{(23)}$.

Nesse contexto, a monitoração ácido-básico é uma intervenção importante, dentro da Classe Controle Eletrolítico e Ácido-Básico. Dentre as atividades possíveis, destaca-se determinar os níveis da gasometria arterial, cujos dados obtidos, como a incapacidade do sistema respiratório em captar oxigênio e/ou remover dióxido de carbono do sangue, ajudam a definir e/ou ajustar o tipo de suporte ventilatório a ser instituído em pacientes com alterações do padrão respiratório, bem como a elaboração de novos diagnósticos de enfermagem relacionados à função respiratória ${ }^{(36)}$.

Ao considerar o "domínio segurança", o foco do cuidado prestado pela(o) enfermeira(o) está no controle de infecções que pode emergir durante a permanência da criança no ambiente hospitalar, uma vez que a admissão em unidade pediátrica é necessária quando a criança é acometida pela forma grave da COVID-19, realidade compartilhada no estudo realizado na Espanha, que descreve o tratamento pediátrico para o Coronavírus 2019, onde 60\% das crianças evoluíram para hospitalização ${ }^{(20)}$. Com base nesse contexto, estudo acerca do tratamento de crianças com COVID-19, em uma unidade de terapia intensiva 
pediátrica em Cingapura, propôs medidas especiais para otimizar o controle da exposição da criança, do familiar e do profissional e minimizar a disseminação do vírus e o desenvolvimento da doença, tendo em vista também a contaminação por outras infecções nosocomiais ${ }^{(37)}$. Entre as medidas, foram citadas a permanência de apenas um profissional de saúde com cada criança, geralmente para prestar os cuidados de enfermagem, que deverá fazer uso de máscara cirúrgica e permanecer no quarto com a criança em tempo integral; preferencialmente que as salas sejam de pressão negativa e possuam banheiros privativos, para minimizar o movimento de pais e filhos na ala pediátrica, além de adotar ações de higiene e a utilização dos demais equipamentos de proteção individual ${ }^{(37)}$.

Ainda em relação ao domínio segurança, as três intervenções: "controle ambiental: segurança", "monitoração dos sinais vitais" e "supervisão", são consideradas pela NIC como essenciais para a especialidade enfermagem pediátrica $^{(23)}$. Nesse caso, o controle ambiental está voltado para o monitoramento e a manipulação do ambiente físico para a promoção da segurança. A monitoração dos sinais vitais é importante para a criança que apresenta infecção respiratória, por colaborar na melhora clínica, por vezes temporária, quando em suporte ventilatório, como demonstra o estudo que buscou descrever o uso da cânula nasal de alto fluxo em crianças no serviço de emergência pediátrica ${ }^{(38)}$. Em se tratando de crianças em estado de gravidade, a monitoração dos sinais vitais e a supervisão para obter dados do paciente para a tomada de decisão clínica ajudam a equipe de enfermagem na observação da evolução clínica da doença, o que requer a continuidade das práticas de cuidado já utilizadas quando esta evolui de forma positiva ou em mudança diante de respostas fisiológicas negativas da criança.

O "domínio comportamental" foi contemplado com ações voltadas para o processo de orientação ao paciente, e sempre que possível, aos pais/cuidadores em relação aos processos da doença, à situação prevista, bem como a realização de cuidados que ajudam no processo de tratamento da criança. As práticas de orientação de pais/cuidadores de crianças hospitalizadas são apontadas em estudos como de suma importância, visto que estes são considerados uma extensão dos profissionais de enfermagem, uma vez que promovem os cuidados recomendados pela equipe de enfermagem, facilitando a adesão da criança ao tratamento ${ }^{(39)}$. Além disso, o envolvimento da família nesse cuidado facilita o estabelecimento de comunicação e o relacionamento entre a equipe, a criança hospitalizada e o familiar cuidador.

Esse envolvimento entre família e enfermeira(o) facilita a percepção profissional acerca dos sinais de desgaste emocional, e junto aos demais membros da equipe, contribui para o desenvolvimento de estratégias para diminuir a ansiedade que emerge diante da hospitalização das crianças, principalmente quando a causa é uma doença grave, como pode acontecer na COVID19. Sendo assim, a intervenção apoio emocional é considerada pela NIC como essencial para a enfermagem pediátrica ${ }^{(23)}$. De acordo com a literatura científica, a hospitalização de uma criança em estado grave, associada às incertezas de cura, desponta nos familiares sentimento de tristeza e angústia, acarretando em sintomas psicológicos como a ansiedade ${ }^{(40)}$. Diante desse contexto, a(o) enfermeira(o) junto a outros profissionais de saúde pode atuar de forma integrada para diminuição desses sentimentos oferecendo suporte emocional. Estudo realizado em São Paulo, com 20 acompanhantes de crianças hospitalizadas, revelou que o apoio psicoemocional por meio de conversas, conselhos e esclarecimentos quanto ao estado de saúde da criança amenizou o sofrimento durante a hospitalização e o tratamento da criança ${ }^{(41)}$.

Outro aspecto é abordado no "domínio familiar", cuja intervenção de enfermagem foi direcionada à promoção dos valores, interesses e metas da família. Nessa perspectiva, torna-se fundamental proporcionar os conhecimentos necessários aos familiares, utilizar estratégias como ligações telefônicas consentidas pelos cuidadores e responsáveis com a finalidade de orientação e monitoramento do quadro da criança, 
como reforça estudo que buscou contextualizar as experiências vividas no enfrentamento da pandemia da COVID-19, por uma equipe multiprofissional de residentes em Saúde Mental Infanto-juvenil, em um Centro Especializado de Reabilitação do Distrito Federal ${ }^{(42)}$. Além disso, é indispensável respeitar e apoiar mecanismos adaptativos de enfrentamento usados pela família. Como visto, o acometimento respiratório grave na criança com COVID-19 extrapola as dimensões clínicas, e reforça a importância do seguimento dos cuidados necessários ao seu bem-estar biopsicossocial no contexto familiar após a alta.

Contudo, o presente estudo limita-se a diagnósticos e intervenções de enfermagem a crianças com sinais respiratórios de gravidade em COVID-19 mais citados na literatura, porém, outros sinais/sintomas de gravidade devem ser considerados para a integralidade do cuidado em enfermagem a esse público.

\section{Conclusão}

Mesmo que a maioria das crianças com COVID-19 não evolua para a gravidade, ela requer da enfermagem um cuidado individualizado, efetivo e sistematizado, mediante uma linguagem unificada e universal, para atender as suas necessidades humanas básicas e que contribua no seu processo de recuperação. Assim, a(o) enfermeira(o) precisa se antecipar e estar preparada(o) para tal.

Assim, este estudo pôde contribuir para que a(o) enfermeira(o) compreenda os sinais de gravidade presentes em fluxogramas estabelecidos em suas práticas cotidianas, mesmo que estes não contemplem todos os sinais de gravidade a serem observados na pediatria, mas que o(a) profissional possa, com base nesses instrumentos avaliativos norteadores, identificar na criança com COVID-19, as características definidoras que justifiquem de forma efetiva seus diagnósticos e por conseguinte as intervenções, que contemplem a criança e sua família em todo o contexto das suas reais necessidades.
Fica evidenciada a importância dos 3 diagnósticos e das 24 intervenções de enfermagem apresentados neste estudo, sobretudo pelo fato de que as intervenções voltadas para os diagnósticos requerem, em quase sua totalidade, que sejam executadas pela(o) enfermeira(o) assistencial, o que demonstra a necessidade de formação e respaldo técnico-científico para o planejamento do cuidado à criança com sinais respiratórios de gravidade da COVID-19, principalmente por ser uma doença nova em que o conhecimento ainda está sendo construído.

Tendo em vista a abordagem da atualidade e respeitando o processo metodológico da construção científica para a Enfermagem, a descrição dos diagnósticos e intervenções de enfermagem, ancorados pelas taxonomias NANDA e NIC, respectivamente, apresenta inovação na literatura científica brasileira.

Desse modo, traz visibilidade à profissão, conferindo a cientificidade no cotidiano da assistência de Enfermagem e fortalecendo a articulação do ensino com a pesquisa e com o processo de trabalho da enfermagem, o que reverbera na qualidade e na responsabilidade do profissional nos cuidados pediátricos.

Ademais, para a validação dos diagnósticos e intervenções de enfermagem para a criança com COVID-19, recomendamos outros estudos com abordagem observacional, estudos de caso e ensaios clínicos.

\section{Colaborações:}

1 - Concepção, projeto, análise e interpretação dos dados: Renise Bastos Farias Dias, Luana Cavalcante Costa Ferraz, Nirliane Ribeiro Barbosa, Rita de Cássia Batista de Oliveira Peixoto, Maria Betânia Monteiro de Farias, Larissa Tenório Andrade Correia e Andrey Ferreira da Silva;

2 - Redação do artigo e revisão crítica relevante do conteúdo intelectual: Renise Bastos Farias Dias, Luana Cavalcante Costa Ferraz, Nirliane Ribeiro Barbosa, Rita de Cássia Batista de Oliveira Peixoto, Maria Betânia Monteiro de 
Farias, Larissa Tenório Andrade Correia e Andrey Ferreira da Silva;

3 - Aprovação final da versão a ser publicada: Renise Bastos Farias Dias, Luana Cavalcante Costa Ferraz, Nirliane Ribeiro Barbosa, Rita de Cássia Batista de Oliveira Peixoto, Maria Betânia Monteiro de Farias, Larissa Tenório Andrade Correia e Andrey Ferreira da Silva.

\section{Referências}

1. Gorbalenya A, Baker S, Baric R. The species Severe acute respiratory syndrome-related coronavirus: classifying 2019-nCoV and naming it SARS-CoV-2. Nat Microbiol. 2020;5(4):536-44. DOI: https://doi.org/10.1038/s41564-020-0695-Z

2. Benvenuto D, Giovanetti M, Salemi M, Prosperi M, Flora C, Junior Alcantara LC, et al. The global spread of 2019-nCoV: a molecular evolutionary analysis. PathogGlob Health. 2020;114(2):64-7. DOI: https://doi.org/10.1080/20 477724.2020 .1725339

3. Hassan SA, Sheikh FN, Jamal S, Ezeh JK, Akhtar A. Coronavirus (COVID-19): A Review of Clinical Features, Diagnosis, and Treatment. Cureus. 2020;12(3):e7355. DOI: https://doi.org/ 10.7759 /cureus. 7355

4. Rothan HA, Byrareddy SN. The epidemiology and pathogenesis of coronavirus disease (COVID-19) outbreak. J Autoimmun. 2020;109:102433. DOI: https://doi.org/10.1016/j.jaut.2020.102433

5. Simpson JM, Newburger JW. Multisystem Inflammatory Syndrome in Children in Association with COVID-19. Circulation. 2020;142(5):437-40. DOI: https://doi.org/10.1161/ CIRCULATIONAHA.120.048726

6. Hockenberry MJ, Wilson D, Rodgers CC. WONG - Fundamentos de Enfermagem Pediátrica. 10a ed. Rio de Janeiro: Guanabara Koogan; 2018.

7. Ludvigsson JF. Systematic review of COVID-19 in children shows milder cases and a betterprognosisthanadults. Acta Paediatr. 2020;109(6):1088-95. DOI: https://doi.org/10.1111/ apa. 15270

8. Bialek S, Gierke R, Hughes M, McNamara LA, Pilishvili T, Skoff T. Coronavirus Disease 2019 in Children - United States, February 12-April 2, 2020. MMWR Morb Mortal Wkly Rep. 2020;69(14):422-6. DOI: https://doi.org/10.15585/mmwr.mm6914e4
9. Istituto Superiore di Sanità. Epidemia COVID-19 [Internet]. Rome (IT); 2020 [cited 2020 Jun 22]. Available from: https://www.iss.it/rapporti-covid-19

10. Brasil. Ministério da Saúde. Secretaria de Vigilância em Saúde. Boletim Epidemiológico Especial n. 17: COE COVID-19 [Internet]. Brasília (DF); 2020 [cited 2020 May 18]. Available from: https://www. saude.gov.br/images/pdf/2020/May/29/2020-0525---BEE17---Boletim-do-COE.pdf

11. Kelvin AA, Halperin S. COVID-19 in children: the link in the transmission chain. Lancet Infect Dis. 2020;20(6):633-4. DOI: https://doi.org/10.1016/ S1473-3099(20)30236-X

12. Riphagen S, Gomez X, Gonzalez-Martinez C, Wilkinson N, Theocharis P. Hyper inflammatory shock in children during COVID-19 pandemic. Lancet. 2020 May;395(10237):1607-8. DOI: https:// doi.org/ 10.1016/S0140-6736(20)31094-1

13. Dong Y, Mo X, Hu Y, Qi X, Jiang F, Jiang Z, et al. Epidemiology of COVID-19 Among Children in China. Pediatrics. 2020;145(6):e20200702. DOI: https://doi.org 10.1542/peds.2020-0702

14. Silva NEK, Sancho LG, Figueiredo WS. Entre fluxos e projetos terapêuticos: revisitando as noções de linha do cuidado em saúde e itinerários terapêuticos. Ciênc saúde colet. 2016;21(3):843-52. DOI: https://doi.org/10.1590/1413-81232015213. 08572015

15. Brasil. Ministério da Saúde. Fluxo de Manejo Clínico Pediátrico na Atenção Especializada [Internet]. Brasília (DF); 2020 [cited 2020 Jun 21]. Available from: https://www.saude.ms.gov.br/ wp-content/uploads/2020/03/Fluxo-de-manejoclinico-pedi-trico.pdf

16. Viegas LM, Fernandes AMA, Veiga MDAPLF. Intervenção de enfermagem no estresse do cuidador familiar do idoso com dependência: estudo piloto. Rev baiana enferm. 2018;32:e25244. DOI: https://doi.org/ 10.18471/rbe.v32.25244

17. Mota M, Cunha M, Santos M, Olm Cunha ICK, Alves M, Marques N. Intervenções de enfermagem pré-hospitalar: revisão narrativa. Enferm em Foco. 2020;10(4). DOI: https://doi.org/10.21675/2357707X.2019.v10.n4.2527

18. Conselho Federal de Enfermagem. Resolução COFEN n. 358/2009, de 15 de outubro de 2009. Dispõe sobre a Sistematização da Assistência de Enfermagem e a implementação do Processo de Enfermagem em ambientes, públicos ou privados, em que ocorre o cuidado profissional 
de Enfermagem, e dá outras providências. Brasília (DF); 2009 [cited 2020 Jul 2]. Available from: http:// www.cofen.gov.br/resoluo-cofen-3582009_4384. html

19. Vosgerau DSR, Romanowski JP. Estudos de revisão: implicações conceituais e metodológicas. Rev Diálogo Educ. 2014;14(41):165. DOI: https:// doi.org/10.7213/dialogo.educ.14.041.DS08

20. Tagarro A, Epalza C, Santos M, Sanz-Santaeufemia FJ, Otheo E, Moraleda C, et al. Screening and Severity of Coronavirus Disease 2019 (COVID-19) in Children in Madrid, Spain. JAMA Pediatr. 2020; e201346. DOI: https://doi.org/10.1001/ jamapediatrics.2020.1346

21. DeBiasi RL, Song X, Delaney M, Bell M, Smith K, Pershad J, et al. Severe COVID-19 in Children and Young Adults in the Washington, DC Metropolitan Region. J Pediatr. 2020;223:199-203. DOI: https:// doi.org/10.1016/j.jpeds.2020.05.007

22. Herdman TD, Kamitsuru S. Diagnósticos de enfermagem da NANDA-I: definições e classificação 2018-2020. 11a ed. Porto Alegre: Artmed, 2018.

23. Bulechek GM, Butcher HK, Dochterman JM. Classificação das intervenções de enfermagem (NIC). Soraya Imon de Oliveira, tradutora. 5a ed. Rio de Janeiro: Elsevier; 2010.

24. Souza TH, Nadal JA, Nogueira RJN, Pereira RM, Brandão MB. Clinical manifestations of children with COVID-19: A systematic review. Pediatr Pulmonol. 2020 Jun;55(8):1892-9. DOI: https://doi. org/10.1002/ppul.24885

25. Choi S-H, Kim HW, Kang J-M, Kim DH, Cho EY. Epidemiology and clinical features of coronavirus disease 2019 in children. Clin Exp Pediatr. 2020;63(4):125-32. DOI: https://doi.org/ 10.3345/ cep. 2020.00535

26. Cao Q, Chen Y-C, Chen C-L, Chiu C-H. SARS-CoV-2 infection in children: Transmission dynamics and clinical characteristics. J Formos Med Assoc. 2020;119(3):670-3. DOI: https://doi.org/10.1016/ j.jfma.2020.02.009

27. Sociedade Brasileira de Pediatria. Nota de alerta - Sistematização da assistência de pacientes com COVID-19 no serviço de emergência pediátrica [Internet]. Brasília (DF); 2020 [cited 2020 Jun 21]. Available from: https://www.sbp.com.br/ fileadmin/user_upload/22463c-NA_-Sistematiz_ Assist_Covid-19_Serv_EmergPed.pdf

28. Branco CSN, Pamplona YDAP. Diagnósticos de enfermagem em crianças portadoras de insuficiência renal crônica em tratamento hemodiálico. Rev Enferm Contemp. 2013;2(1). DOI: https://doi.org/10.17267/2317-3378rec.v2i1.235

29. Pascoal LM, Lopes MVO, Chaves DBR, Beltrão BA, Silva VM, Monteiro FPM. Impaired gas exchange: accuracy of defining characteristics in children with acute respiratory infection. Rev Latino-Am Enfermagem. 2015 Jul 3;23(3):491-9. DOI: 10.1590/ 0104-1169.0269.2581

30. Prado PR, Bettencourt ARC, Lopes JL. Defining characteristics and related factors of the nursing diagnosis for ineffective breathing pattern. Rev Bras Enferm. 2019 Feb;72(1):221-30. DOI: https:// doi.org 10.1590/0034-7167-2018-0061

31. Canto DF, Almeida MA. Resultados de enfermagem para padrão respiratório ineficaz e ventilação espontânea prejudicada em terapia intensiva. Rev Gaúcha Enferm. 2013 dez;34(4):137-45. DOI: https://doi.org/10.1590/\$1983-14472013000400018

32. Zhang L, Peres TG, Silva MVF, Camargos P. What we know so far about Coronavirus Disease 2019 in children: A meta-analysis of 551 laboratory-confirmed cases. Pediatr Pulmonol. 2020;55(8):2115-27. DOI: https://doi.org/10.1002/ ppul.24869

33. Oualha M, Bendavid M, Berteloot L, Corsia A, Lesage $\mathrm{F}$, Vedrenne $\mathrm{M}$, et al. Severe and fatal forms of COVID-19 in children. Arch Pédiatrie. 2020;27(5):235-8. DOI: https://doi.org/10.1016/j. arcped.2020.05.010

34. Giacomello KJ, Melo LL. The meaning of the care of hospitalized children: experiences of nursing professionals. Rev Bras Enferm. 2019;72(Suppl 3):251-8. DOI: https://doi.org/ 10.1590/0034-7167-2018-0597

35. Li Y, Bai W, Hashikawa T. The neuro invasive potential of SARS $\square$ CoV2 may play a role in the respiratory failure of COVID-19 patients. J Med Virol. 2020 Jun 11;92(6):552-5. DOI: https://doi. org/10.1002/jmv.25728

36. Rolim LR, Melo EM, Frota NM, Almeida NG, Barbosa IV, Caetano JA. Conhecimento do profissional de enfermagem da unidade de terapia intensiva sobre gasometria arterial. Rev enferm UFPE line [Internet]. 2013 [cited 2020 Jul 2];7(3):713-21. Available from: https://periodicos. ufpe.br/revistas/revistaenfermagem/article/view/ 10284/10930

37. Thampi S, Yap A, Fan L, Ong J. Special considerations for the management of COVID-19 
pediatric patients in the operating room and pediatric intensive care unit in a tertiary hospital in Singapore. Pediatr Anesth. 2020;30(6):642-6. DOI: https://doi.org/10.1111/pan.13863

38. Slain KN, Shein SL, Rotta AT. The use of high-flow nasal cannula in the pediatric emergency department. J Pediatr (Rio J). 2017 Nov;93 (Suppl 1):36-45. DOI: https://doi. org/10.1016/ j.jped.2017.06.006

39. Azevêdo AVS, Lançoni Júnior AC, Crepaldi MA. Interação equipe de enfermagem, família, e criança hospitalizada: revisão integrativa. Ciênc saúde coletiva. 2017;22(11):3653-66. DOI: https:// doi.org/10.1590/1413-812320172211.26362015

40. Costa TS, Morais AC. A hospitalização infantil: vivência de crianças a partir de representações gráficas. Rev enferm UFPE on line. 2016;11(1):358-67. DOI: https://doi. org/10.5205/1981-8963-v11i1a11916p358-367-2017
41. Kozan L, Wanderbroocke AC, Polli GM. Apoio social entre acompanhantes de crianças hospitalizadas em uma unidade de hematopediatria. Psicol hosp. [Internet]. 2016 [cited 2020 Jun 14];14(1):53-78. Available from: http://pepsic.bvsalud.org/scielo.php?script=sci_ arttext\&pid=S1677-74092016000100004\&lng=pt\&n $\mathrm{rm}=\mathrm{iso}$

42. Santos M, Parente B, Lima G, Mariano D, Carvalho M. Saúde mental de crianças e seus cuidadores diante da pandemia da COVID-19. Health Residencies Journal. 2020 [cited 2020 Jun 21];1(5). Available from: https://escsresidencias.emnuvens. com.br/hrj/article/view/14

Recebido: 22 de Junho de 2020

Aprovado: 13 de Julho de 2020

Publicado: 26 de novembro de 2020

A Revista Baiana de Enfermagem utiliza a Licença Creative Commons - Atribuição-NãoComercial 4.0 Internacional. https://creativecommons.org/licenses/by-nc/4.0/

Este artigo é de acesso aberto distribuído sob os termos da Licença Creative Commons (CC BY-NC). Esta licença permite que outros remixem, adaptem e criem a partir do seu trabalho para fins não comerciais. Embora os novos trabalhos tenham de lhe atribuir o devido crédito e não possam ser usados para fins comerciais, os usuários não têm de licenciar esses trabalhos derivados sob os mesmos termos. 